\title{
Probing the Debye spectrum in glasses using small system sizes
}

\author{
Matteo Paoluzzi $\odot, 1,2,3,{ }^{*}$ Luca Angelani, ${ }^{2,3}$ Giorgio Parisi, ${ }^{3,4,5}$ and Giancarlo Ruocco ${ }^{3,6}$ \\ ${ }^{1}$ Departament de Física de la Matèria Condensada, Universitat de Barcelona, Carrer de Martí i Franquès 1, 08028 Barcelona, Spain \\ ${ }^{2}$ ISC-CNR, Institute for Complex Systems, Piazzale A. Moro 2, I-00185 Rome, Italy \\ ${ }^{3}$ Dipartimento di Fisica, Sapienza Università di Roma, Piazzale A. Moro 2, I-00185 Rome, Italy \\ ${ }^{4}$ Nanotec-CNR, UOS Rome, Piazzale A. Moro 2, I-00185 Rome, Italy \\ ${ }^{5}$ INFN-Sezione di Roma 1, Piazzale A. Moro 2, I-00185 Rome, Italy \\ ${ }^{6}$ Center for Life Nano Science, Istituto Italiano di Tecnologia, Viale Regina Elena 291, I-00161 Rome, Italy
}

(Received 17 January 2020; accepted 27 October 2020; published 18 November 2020)

\begin{abstract}
We investigate the low-frequency spectrum in a three-dimensional model of structural glass focusing on small system sizes, and using different observables, i.e., the density of states $D(\omega)$, the cumulative of the density of states $F(\omega)$, and the dynamical structure factor $S(q, \omega)$ in the harmonic approximation. When the glass is obtained by an instantaneous quench from high temperatures, we show that extended "phonon-like" modes always populate the low-energy spectrum. Looking at the properties of the dynamical structure factor $S(q, \omega)$, we observe that in agreement with early studies of Lennard-Jones glasses [V. Mazzacurati, G. Ruocco, and M. Sampoli, Europhys. Lett. 34, 681 (1996)], there are still extended modes below the lowest resonant peak. These modes give rise to a plateau in the $S(q, \omega)$ for $\omega \rightarrow 0$. This result indicates that the low-energy spectrum of extended modes in glasses can be probed using small system sizes and performing instantaneous quench from high parental temperatures. As we recently observed [M. Paoluzzi, L. Angelani, G. Parisi, and G. Ruocco, Phys. Rev. Lett. 123, 155502 (2019)], the situation changes when the glassy configuration is obtained by an instantaneous quench from lower temperatures. The former protocol suppresses extended modes below the lowest resonant peak emphasizing the localized modes with $D(\omega) \sim \omega^{4}$.
\end{abstract}

DOI: 10.1103/PhysRevResearch.2.043248

\section{INTRODUCTION}

The low-frequency portion of the vibrational density of states (DOS) $D(\omega)$ in topologically disordered systems is dominated by Goldstone modes (spatially extended quasiphonon modes) whose DOS is proportional to $\omega^{d-1}$, where $d$ is the spatial dimension. Overwhelmed by these phonon modes, other elusive excitations are expected to exist. Besides the two-level systems, present only in quantum systems and showing their existence only at very low temperature [1], localized excitations with a $\omega^{4}$ DOS in any dimension are supposed to exist as a consequence of the marginal stability at the spectrum edge of the dynamical matrix $[2,3]$.

Recently different methods have been proposed to highlight the localized modes [4-9]. In Ref. [5], the authors proposed to simulate systems with small enough size $L$ in order to keep "room" at frequencies below the lowest resonant mode [at frequency $\omega_{0}=v_{T}(2 \pi / L)$, where $v_{T}$ is the transverse sound velocity], thus assuming that below $\omega_{0}$ all the

\footnotetext{
*matteopaoluzzi@ub.edu

Published by the American Physical Society under the terms of the Creative Commons Attribution 4.0 International license. Further distribution of this work must maintain attribution to the author(s) and the published article's title, journal citation, and DOI.
}

modes were localized [5]. With these methods, they claimed to always observe an $\omega^{4}$ density of states below $\omega_{0}$.

An alternative approach has been used by us [7,9]. In a first work [7], we pinned some of the particles during the search of the inherent structures [10-14], in such a way to kill the extended modes, i.e., collective excitations that are phonon-like, leaving almost unperturbed the localized ones that were able to vibrate in between the pinned particles. In our three-dimensional system, we observed a progressive transition of the DOS from $\omega^{2}$ (no pinning) toward $\omega^{4}$ (high pinning fraction) [7]. In a second work [9], building on the heterogenous elasticity theory [15-18] and on the existence of larger and larger correlation length on decreasing the temperature toward the dynamical transition temperature $T_{d}$ in supercooled liquids [19-26], we use the parental temperature instead of the pinning fraction as a control parameter. The parental temperature is the temperature at which the liquid is equilibrated before to start the zero-temperature instantaneous quenching procedure. We have observed a continuous transition of the DOS from $\omega^{2}$ (high parental temperature, no heterogeneity) toward $\omega^{4}$ (low parental temperature, large heterogeneity).

In all these studies we used "small systems," i.e., up to $N=20^{3}$, where-as we found-many excitations have frequencies below the lowest resonant mode. Nonetheless, we observed both $\omega^{4}$ (associated with the localized modes) and $\omega^{2}$ (associated with the $d=3$ extended modes) well below the lowest resonant mode. In this work, focusing our attention 
on small system sizes, we show that, in agreement with our previous results [9], annealing protocols rather than the choice of small system sizes are the ingredient for observing the $D(\omega) \sim \omega^{4}$ power law.

All these observations lead to the conclusion that unless one introduces an annealing protocol [27], it is useless to analyze small systems if one aims to emphasize the localized modes. The latter show up only in strongly heterogeneous systems.

Considering small system sizes, in the present paper, we show that different observables such as the DOS $D(\omega)$, the cumulative $F(\omega)$, and the dynamical structure factor $S(q, \omega)$ suggest that the spectrum below the lowest resonant mode still follows Debye's law in homogenous glasses, i.e., glassy configurations obtained after an instantaneous quench from $T=\infty$. We thus connect the spectral shape of the dynamical structure factor $S(q, \omega)$ at low frequency with the behavior of $D(\omega)$.

These findings make a connection with the glass stability issue. Indeed, strong heterogeneity could be the consequence of ill thermalization (unstable glass). Also, this study points out that on the contrary, it is very useful to consider small system sizes with respect to large ones if one aims to study the Debye law at low frequencies. This is because of the cost of diagonalizing a matrix of size $N$ scales with $N^{3}$. Through the diagonalization of the dynamical matrix one computes $3 N$ eigenvalues; the same information can be obtained considering $n$ smaller systems each one of size $M=N / n$ with a time cost that scales as $N^{3} / n^{2}<N^{3}$.

Moreover, our results show that when the parental configuration is disordered but homogeneous, i.e., it is taken deep in the liquid phase, extended modes populate the low-frequency spectrum below the lowest transverse mode. Configurations taken at parental temperatures close to the dynamic transition, i.e., where dynamical heterogeneities proliferate, maintain almost untouched the resonant mode. However, they lose extended modes at lower frequencies and thus the only lowenergy excitations below $\omega_{0}$, i.e., the lowest resonant modes, are soft-localized modes.

\section{MODEL AND METHODS}

The microscopic model consists of a 50 : 50 binary mixture composed of small and large particles labeled with $\mathrm{A}$ and $\mathrm{B}$, respectively. The system is confined in a cubic box of side $L$ with periodic boundary conditions. Two particles interact through a pure repulsive pairwise potential $[28,29]$. The total number of particles reads $N=N_{A}+N_{B}$ and the corresponding density $\rho=N / L^{3}$ is fixed to $\rho=1$; i.e., the side of the box is $L=N^{1 / 3}$. The radii are $\sigma_{A}$ and $\sigma_{B}$ with $\sigma_{A} / \sigma_{B}=1.2$ and $\sigma_{A}+\sigma_{B} \equiv \sigma=1$ [29]. Indicating with $\mathbf{r}_{i}$ the position of the particle $i$, with $i=1, \ldots, N$, two particles $i, j$ interact via the potential

$$
\phi\left(r_{i j}\right)=\epsilon\left[\left(\sigma_{i}+\sigma_{j}\right) / r_{i j}\right]^{12}+k_{0}+k_{2} r_{i j}^{2},
$$

where $r_{i j} \equiv\left|\mathbf{r}_{i}-\mathbf{r}_{j}\right|$. We impose a cutoff to the potential at $r_{c}=\sqrt{3} \sigma$ in a way that $\phi(r)=0$ for $r>r_{c}$. The coefficients $k_{0}$ and $k_{2}$ guarantee continuity to $\phi(r)$ up to the first derivative at $r=r_{c}$. Details about numerical simulations of the model can be found in Refs. [7,9].

\section{A. Generating equilibrium configurations}

Equilibrium configurations have been obtained performing Brownian dynamics at temperature $T$. As temperature decreases toward $T_{d}$, we have introduced swap Monte Carlo moves for improving the thermalization [29]. Details about the dynamics can be found in Ref. [9]. We thus consider the pure relaxation dynamics of the system coupled to a thermal bath at temperature $T$ in the overdamped regime. Every $2 \times 10^{3}$ time steps, we propose an update of the system through standard swap moves [9]. In this work we focus our attention on system sizes $N=12^{3}$ and $N_{s} \in[200,719]$ number of independent samples. In the following, we report all quantities in reduced units considering $\sigma=\epsilon=\mu=1$, with $\mu$ the particle mobility. The number density is $\rho=1$ and $T_{d} \simeq 0.185$.

\section{B. Inherent structures and the density of states}

After thermalization, we compute the corresponding inherent structures through the limited-memory Broyden-FletcherGoldfarb-Shanno algorithm [30]. Let $\mathbf{r}$ be a configuration of the system, i.e., $\mathbf{r} \equiv\left(\mathbf{r}_{1}, \ldots, \mathbf{r}_{N}\right)$. The mechanical energy of the configuration $\mathbf{r}$ is

$$
E[\mathbf{r}]=\sum_{i<j} \phi\left(r_{i j}\right)
$$

We indicate with $\mathbf{r}^{0} \equiv\left(\mathbf{r}_{1}^{0}, \ldots, \mathbf{r}_{N}^{0}\right)$ configurations that correspond to one of the many local minima of $E[\mathbf{r}]$.

The spectrum of the harmonic oscillations around $\mathbf{r}^{0}$ is then obtained considering a perturbed configuration $\mathbf{r}=\mathbf{r}^{0}+\delta \boldsymbol{r}$. The mechanical energy now reads

$$
E[\boldsymbol{r}]=E\left[\mathbf{r}^{0}\right]+\Delta E
$$

with

$$
\Delta E \equiv \frac{1}{2} \sum_{i, j} \sum_{\mu \nu} \delta r_{i}^{\mu} M_{i j}^{\mu v} \delta r_{j}^{\nu},
$$

where $M_{i j}^{\mu \nu}$ indicates the elements of the Hessian matrix. Latin indices $i, j=1, \ldots, N$ indicate the particles and Greek symbols $v, \mu=1, \ldots, 3$ the Cartesian coordinates. We have computed all the $3 N$ eigenvalues $\lambda_{\kappa}=\omega_{\kappa}^{2}$, with $\kappa=$ $1, \ldots, 3 N$, using the GNU Scientific Library (GSL) for sizes up to $N=12^{3}$.

The density of states is defined as

$$
D(\omega)=\mathcal{N}^{-1} \sum_{\kappa} \delta\left(\omega-\omega_{\kappa}\right)
$$

with $\mathcal{N}$ the number of nonzero modes, that is, $3 N-3$ for translationally invariant systems. Another useful observable for studying the low-frequency spectrum is the cumulative distribution $F(\omega)$ that is defined as

$$
F(\omega)=\int_{0}^{\omega} d \omega^{\prime} D\left(\omega^{\prime}\right) .
$$

The main advantage of using $F(\omega)$ instead of $D(\omega)$ is that $F(\omega)$ does not require any binning procedure for the estimation of the properties of the DOS from numerical eigenvalues; i.e., one does not have to compute any histogram for determining $F(\omega)$, because, after performing the integration of delta 
functions $\delta\left(\omega-\omega_{\kappa}\right)$, we immediately realize that the only information one needs is the list of eigenvalues sorted from the smallest to the largest. In the present work, we will compare the results obtained from the direct computation of (5) with those provided by $F(\omega)$. In particular, we will show that while $D(\omega)$ can lead to misleading results, $F(\omega)$ turns out to be a more suitable observable.

Finally, the measure of how the mode of frequency $\omega$ is extended or localized can be estimated through the inverse of the participation ratio $\mathcal{R}$ [31], that is,

$$
\mathcal{R}=\frac{\sum_{i}\left|\mathbf{e}_{i}(\omega)\right|^{4}}{\left[\sum_{i}\left|\mathbf{e}_{i}(\omega)\right|^{2}\right]^{2}},
$$

with $\mathbf{e}_{i}(\omega)$ indicating the eigenvectors of the Hessian matrix. In particular, we will investigate through the probability distribution function $\mathcal{P}(\mathcal{R})$ the localization properties of modes populating the low-frequency sector of the vibrational spectrum. $\mathcal{P}(\mathcal{R})$ is computed considering modes below or around a threshold frequency $\omega_{t h}$.

\section{Computation of the dynamical structure factor $S(q, \omega)$}

The knowledge of normal modes allows us to compute the dynamic structure factor $S(q, \omega)$ in the harmonic approximation [32]. In this approximation the actual interactions have been replaced by harmonic interactions characterized by the set of $3 N$ eigenfrequencies $\omega_{\lambda}^{2}$ and the corresponding eigenvectors $\mathbf{e}_{i}(\lambda)$, i.e., the eigenvector of the mode labeled by $\lambda$. The structure factor $S(q, \omega)$ in the harmonic approximation reads

$$
S_{T, L}(q, \omega)=\frac{k_{B} T}{N} q^{2} \sum_{\lambda}\left|\mathrm{f}_{T, L}(q, \lambda)\right|^{2} \frac{\delta\left(\omega^{2}-\omega_{\lambda}^{2}\right)}{\omega},
$$

where the longitudinal and transversal components, $S_{L}$ and $S_{T}$, respectively, are given by the following expressions:

$$
\begin{aligned}
\mathrm{f}_{L}(q, \lambda) & \equiv \sum_{i} \frac{\mathbf{q}}{q} \cdot \mathbf{e}_{i}(\lambda) e^{i \mathbf{q} \cdot \mathbf{r}_{i}}, \\
\mathbf{f}_{T}(q, \lambda) & \equiv \sum_{i} \frac{\mathbf{q}}{q} \times \mathbf{e}_{i}(\lambda) e^{i \mathbf{q} \cdot \mathbf{r}_{i}} .
\end{aligned}
$$

The wave vectors $\mathbf{q}=\left(q_{x}, q_{y}, q_{z}\right)$ of modulus $q=|\mathbf{q}|$ satisfy the periodic boundary condition imposed to the system. We thus have a minimum wave vector of modulus $q=\frac{2 \pi}{L}$ and a generic $\mathbf{q}$ results in being written as $\mathbf{q}=q_{\min }\left(n_{x}, n_{y}, n_{z}\right)$ with $n_{x, y, z}$ three independent integer numbers.

\section{DENSITY OF STATES}

In this section we discuss the properties of the density of states $D(\omega)$ defined through Eq. (5). We will show that extended modes populate the low-frequency spectrum no matter how small the system size is. $D(\omega)$ results in being bounded at low frequencies by two power laws, i.e., $\omega^{4} \leqslant D(\omega) \leqslant \omega^{2}$. This bound is undetectable in linear scale, where $D(\omega)$ appears to be $\sim \omega^{2}$ within the statistical uncertainties. We will show that the situation changes at low parental temperature; in particular Debye's spectrum disappears for $T \rightarrow T_{d}$, with $T_{d}$ the dynamical temperature.
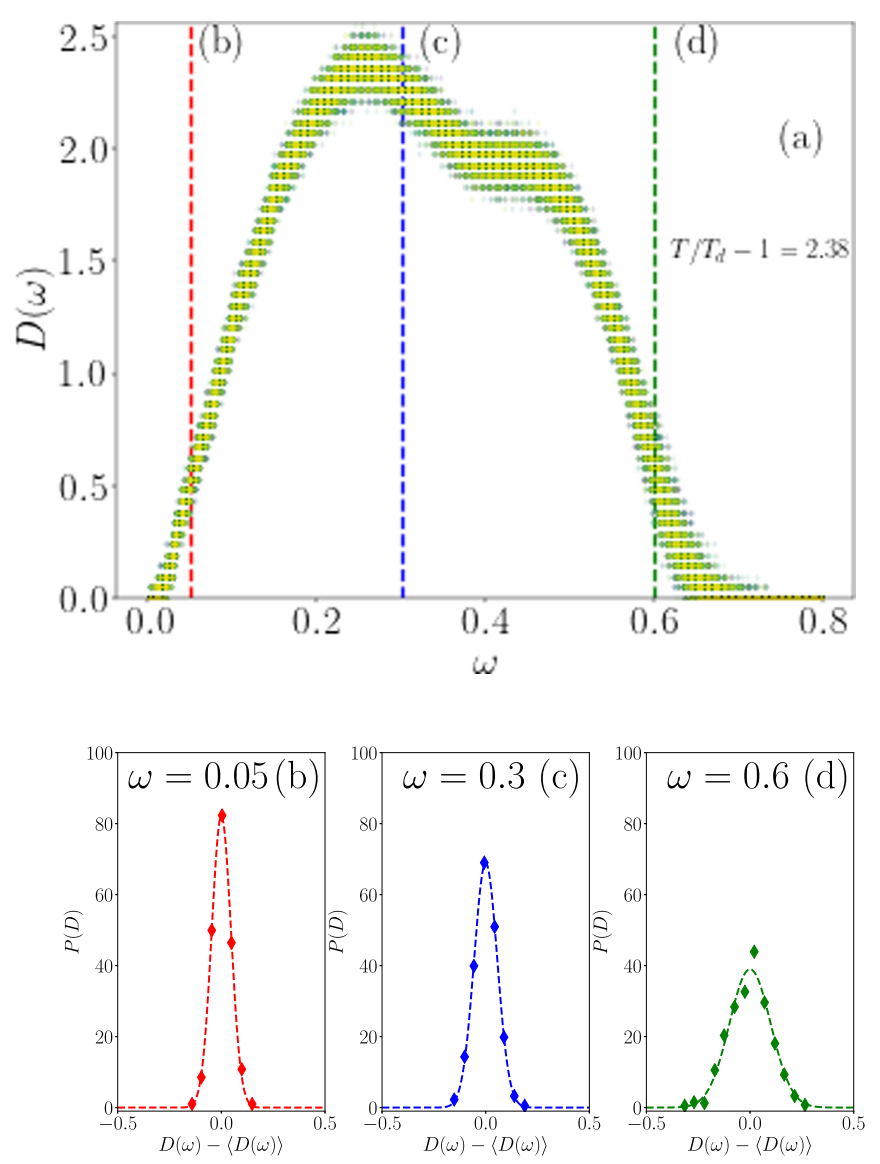

FIG. 1. (a) Density of states $D(\omega)$. The system is composed of $N=12^{3}$ particles, and the inherent structures have been obtained by quenching after the equilibration of systems at high parental temperatures, i.e., $T / T_{d}=2.38$. Different colors are different samples. Here we show 600 samples. Panels (b), (c), and (d): Sample-tosample fluctuations of $D(\omega)$ at three representative frequencies, i.e., $\omega=0.05,0.3,0.6$, from left to right. Symbols are the distribution of values of $D(\omega)$ measured at a given value of frequency $\omega$. The dashed line is the fit with a Gaussian distribution.

\section{A. Debye spectrum at high parental temperature $T \gg T_{d}$}

We first consider configurations thermalized at high parental temperature, i.e., well above the dynamical transition temperature of the model $T_{d}$. Figure 1 shows the corresponding density of states $D(\omega)$. Lower panels highlight sample-to-sample fluctuations at three representative frequencies, i.e., $\omega=0.05,0.3,0.6$. Dashed curves are fits to a Gaussian distribution that turns out to capture the distribution $P(D)$ around its mean value. In Fig. 2, we focus our attention at low frequencies. Our data analysis is consistent with Debye's law below the lowest resonant peak at $\omega_{0} \sim 0.035$. Moreover, above $\omega_{0}$, i.e., $\omega \geqslant 0.04$, data seem to collapse again on Debye's law.

Now, to gain insight into low-energy excitations, we will look at the low-frequency spectrum in more detail. The results are provided in the inset of Fig. 2, where $D(\omega)$ is shown in both linear (main panel) and double logarithmic scale (inset). Different colors refer to linear (red circles) and logarithmic (blue diamonds) data binning. Dashed and dotted lines are the two scalings, $D(\omega) \sim \omega^{2}$ and $D(\omega) \sim \omega^{4}$, respectively. 


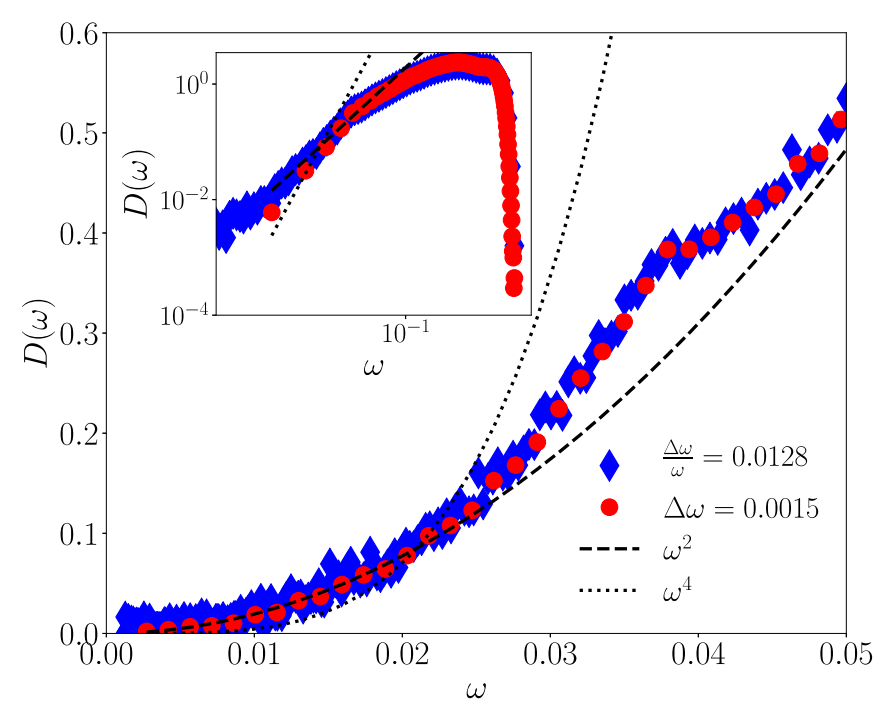

FIG. 2. Density of states $D(\omega)$ at high parental temperature at low frequencies for system size $N=12^{3}$. Red circles, linear data binning; blue diamonds, logarithmic data binning. The dashed line is the Debye scaling $D(\omega) \sim \omega^{2}$. The dotted curve is the $\omega^{4}$ scaling. Both have been obtained by fitting the data below $\omega=0.025$. Inset: $D(\omega)$ in double logarithmic scale, $\Delta \omega=0.007$ (red circles) and $\frac{\Delta \omega}{\omega}=0.066$ (blue diamonds).

\section{B. Deviations from Debye spectrum at low parental temperatures $T \rightarrow T_{d}$}

Here we consider the density of states $D(\omega)$ of inherent structures obtained from configurations thermalized at a parental temperature $T$ close to the dynamical transition temperature of the considered model from above. In Ref. [9] we showed that the slope of the cumulative function $F(\omega)$ continuously changes from Debye to non-Debye, i.e., from 3 to 5 , as the temperature decreases toward $T_{d}$.

This finding is confirmed in Fig. 3 where we show $D(\omega)$ in linear scale and log scale (inset). As one can see, although $D(\omega)$ develops a fat tail below $\omega_{0}$ (inset), the Debye spectrum results in being suppressed in favor of an $\omega^{4}$ power law at low frequencies. This finding confirms what we have observed in Ref. [9]. It is worth noting that the annealing procedure removes any $\omega^{2}$ contribution below the resonant peak that remains at $\omega=0.04$.

\section{The cumulative density of states $F(\omega)$}

In the previous section, we showed that a genuine $\omega^{4}$ scaling in the low-frequency regime in $D(\omega)$ can be observed at low parental temperatures. Moreover, for high parental temperatures, $D(\omega)$ shows a clear Debye scaling at low frequencies, even below the resonant peak at $\omega_{0}$, no matter how small the size of the system is.

Here we show the same behavior also in the cumulative density of states $F(\omega)$ that is defined through Eq. (6). Since $D(\omega)$ is a sum of delta functions, for computing $F(\omega)$ one has just to sort the eigenfrequencies of the dynamical matrix from the lowest to the largest. In this way, the result does not depend on any particular choice made for the data binning. It is worth noting that $F(\omega) \sim \omega^{3}$ corresponds to Debye's scaling

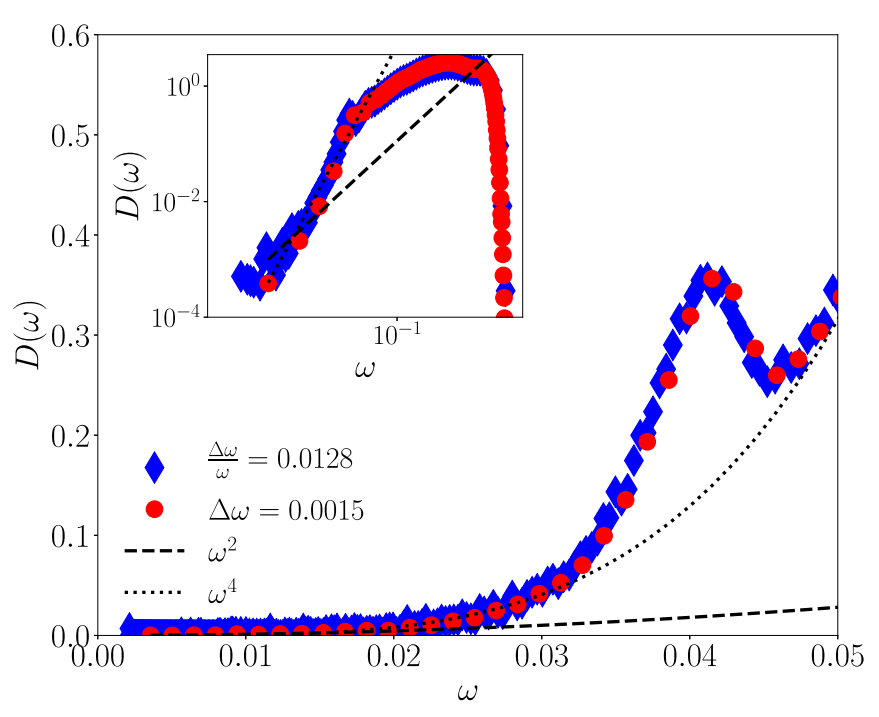

FIG. 3. Density of states $D(\omega)$ at low frequencies (the system size is $N=12^{3}$ ) and parental temperature $T / T_{d}-1=0.01$. The dashed line is the Debye scaling $D(\omega) \sim \omega^{2}$; the dotted line is the $\omega^{4}$ scaling. Both curves have been obtained by fitting the data below $\omega=0.025$. Red circles refer to linear data binning, blue diamonds to logarithmic data binning. Inset: $D(\omega)$ in double logarithmic scale, $\Delta \omega=0.007$ (red circles) and $\frac{\Delta \omega}{\omega}=0.066$ (blue diamonds).

$D(\omega) \sim \omega^{2}$ and $F(\omega) \sim \omega^{5}$ to $D(\omega) \sim \omega^{4}$. The behavior of $F(\omega)$ is reported in Fig. 4. It turns out that samples prepared at high parental temperatures [see panel (a)] follow Debye's scaling below the lowest transverse mode $\omega_{0}$ [32]. On the other hand, as the parental temperature decreases [panel (b)], $F(\omega) \sim \omega^{5}$ below $\omega_{0}$.

To better quantify the properties of these modes, we have computed the probability distribution function $\mathcal{P}(\mathcal{R})$. For computing $\mathcal{P}(\mathcal{R})$, we consider only modes that populate a given sector of the spectrum. In this way, we can discriminate between regions where it is more likely to find extended modes and regions that are populated by localized modes. It is worth noting that for performing a careful study of $\mathcal{R}$, one has to look at the scaling of $\mathcal{R}$ as the size of the system changes. However, the position of the transverse peak $\omega_{0}$ shifts at lower frequencies as the size of the system increases. Since we are interested in understanding the localization properties of modes at frequencies lower than $\omega_{0}$, we perform our study at small system sizes.

We focus our attention on two sectors of the low-frequency spectrum. The first sector is below the lowest transverse mode $\omega_{0}$. The second sector is around $\omega_{0}$. Figure 5 reports the results of our analysis. Dashed curves refer to $\mathcal{P}(\mathcal{R})$ computed in the second sector, the red curves refer to high parental temperature, the blue curves close to the dynamical transition. The location of the peak of the distribution slightly changes around the frequency of the lowest transverse mode. Moreover, as expected for phonons, the peak is located at small $\mathcal{R}$ values that are compatible with completely extended modes (for extended modes $\mathcal{R} \sim N^{-1} \sim 10^{-3}$ [31]). In the low-frequency spectrum, i.e., $\mathcal{R}$ computed at frequencies smaller than $\omega_{0}$, the position of the peak shifts toward higher $\mathcal{R}$ values as temperature decreases (in a completely localized mode $\mathcal{R}=1$ ). 

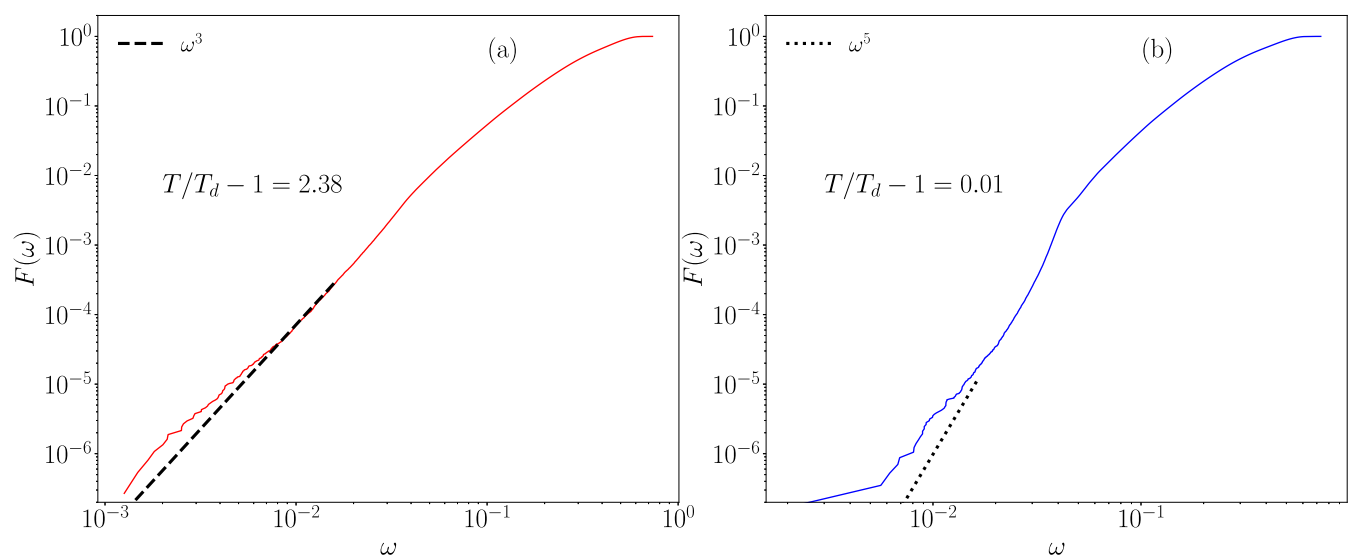

FIG. 4. The cumulative distribution function $F(\omega)$ in double logarithmic scale at high parental temperature (a) and low parental temperature (b). $F(\omega)$ has been obtained by sorting from the smallest to the largest the $N_{s} \times\left(12^{3}-3\right)$ eigenfrequencies, with $N_{s}$ the number of samples. Dashed black line is Debye scaling $\omega^{3}$; dotted black line is non-Debye scaling $\omega^{5}$.

Moreover, at high parental temperatures (red curves), the distributions computed in the two frequency sectors, i.e., around the resonant peak and below it, overlap each other providing further evidence for the presence of extended nonphononic modes in this region of the spectrum.

In the next section, we will show that the behavior of the density of states is consistent with the behavior of the dynamical structure factor $S(q, \omega)$ computed in the harmonic approximation.

\section{DYNAMICAL STRUCTURE FACTOR}

We start our discussion with considering both the transverse and the longitudinal components $S_{T, L}(q, \omega)$ [32-34]. In Fig. 6(a) we show $S_{T, L}\left(q_{\min }, \omega\right)$ as a function of $\omega$, with

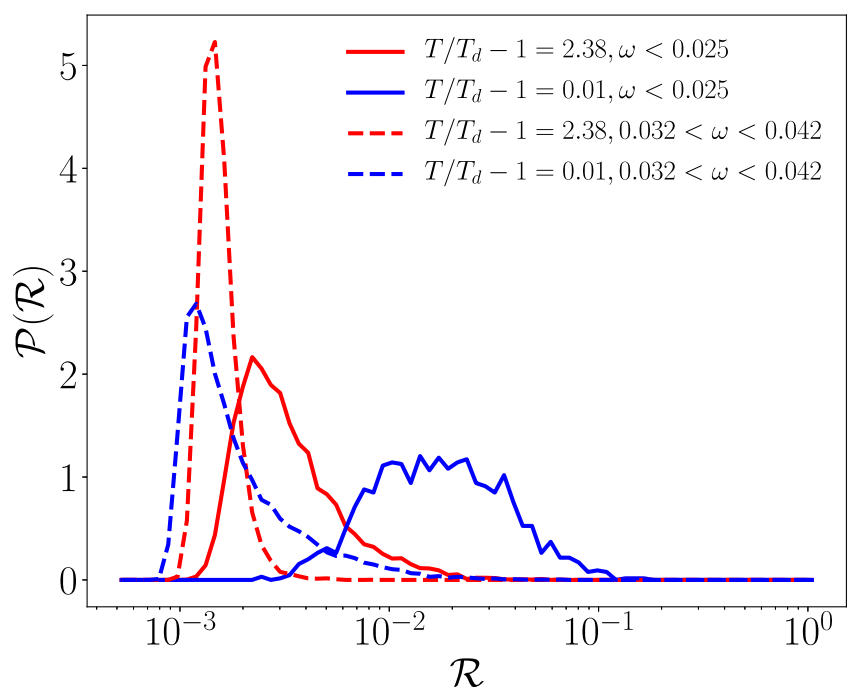

FIG. 5. Probability distribution function $\mathcal{P}(\mathcal{R})$ at high and low parental temperatures (red and blue curves, respectively). $\mathcal{P}(\mathcal{R})$ is computed in two representative regions of the low-frequency spectrum. The first region (dashed curves) is around the transverse mode. The second region (solid curve) is for frequency $\omega$ well below the lowest transverse mode, i.e., $\omega<\omega_{t h}=0.025<\omega_{0} \sim 0.04$. $q_{\min }=\frac{2 \pi}{L} \cdot S_{T, L}\left(q_{\min }, \omega\right)$ are reported for different parental temperatures. Let start with considering the case of high parental temperatures. As one can see, and in agreement with Refs. [32,34], $S_{T, L}(q, \omega)$ show two peculiar features: (i) a broad peak that signals the presence of acoustic-like excitations plus (ii) a plateau that tends to a $q$-independent finite value at zero frequency, i.e., $\lim _{\omega \rightarrow 0} S_{T, L}(q, \omega) \neq 0$.

This behavior is in agreement with the model developed in Ref. [32] where those authors have established the relation

$$
S_{T, L}(q, \omega) \propto \frac{D(\omega)}{\omega^{2}} .
$$

The model predicts that $S_{T, L}(q, \omega)$ is flat at low frequencies for a Debye spectrum. In particular, assuming a low-frequency behavior $D(\omega) \sim \omega^{\alpha}$, one has $S_{T, L}(q, \omega) \sim \omega^{\alpha-2}$. In this picture, a power law for $\omega \rightarrow 0$ in $S_{T, L}(q, \omega)$ reveals deviations from Debye's law. It is worth noting that in the case of a scaling $D(\omega) \sim \omega^{4}$, one has $S_{T, L}(q, \omega \rightarrow 0) \sim \omega^{2}$. We thus have a stringent prediction about the behavior of $S_{T, L}(q, \omega)$ as the parental temperature decreases. Figure 6 suggests that our data are consistent with Eq. (10) providing evidence for $S_{T, L}(q, \omega \rightarrow 0) \sim$ constant at high parental temperatures. Moreover, as the temperature decreases toward $T_{d}$ (same figure), $S_{T, L}(q, \omega)$ shows a different slope at low frequencies with an effective exponent that is (parent) temperature dependent. The same behavior is observed at larger wave numbers, as shown in panel (b) of the same figure, where $S_{T, L}(q, \omega)$ is reported for $q=5 q_{\mathrm{min}}$. Again, at high parental temperatures, $S_{T, L}(q, \omega)$ develops a plateau at small frequencies, i.e., below the resonant peak. The plateau changes into a power-law tail as the temperature changes toward $T_{d}$.

\section{DISCUSSION AND CONCLUSIONS}

Motivated by the upsurge of interest in understanding lowfrequency excitations in glasses, we performed a detailed study of normal modes in a three-dimensional model of glass. In agreement with early studies on the same subject [32], our results provide evidence of the presence of extended modes in the low-frequency spectrum for glassy configurations whose parental configuration belongs to high temperatures. This 

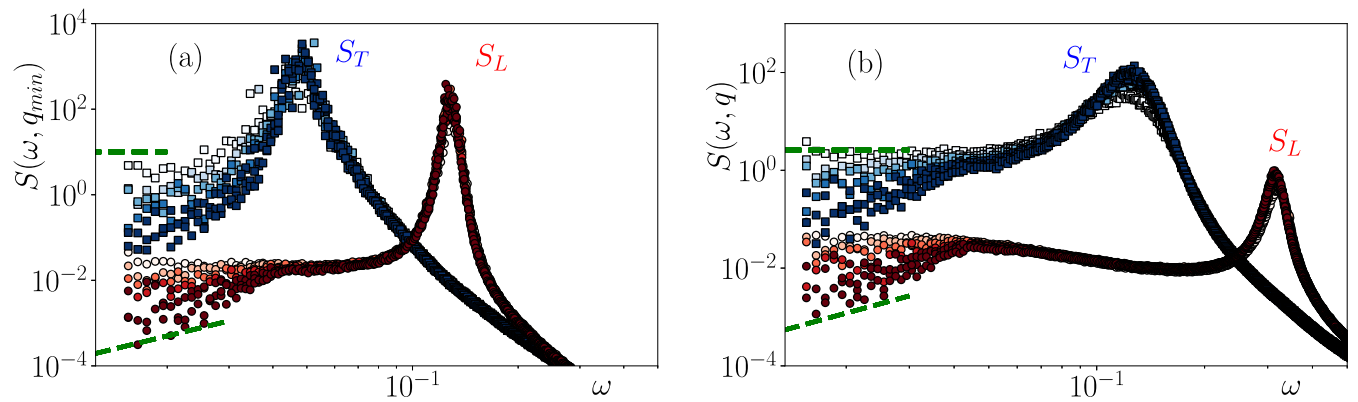

FIG. 6. Dynamical structure factor $S_{T, L}(q, \omega)$ as a function of $\omega$ as the temperature decreases toward $T_{d}$, i.e., $1-T / T_{d}=$ $2.38,0.35,0.28,0.22,0.15,0.08,0.01$ from white to red $\left(S_{L}\right)$ and from white to blue $\left(S_{T}\right)$. In panel (a), $q=q_{\min }$; in panel (b), $q=5 q_{\min }$.

finding is also in agreement with the behavior of the cumulative distribution function of the density of states $F(\omega)$ shown in Refs. [7,9]. In particular, configurations thermalized at high parental temperatures and then instantaneously quenched at $T=0$ for computing the corresponding inherent structures show a Debye spectrum at low frequency, i.e., at frequencies below the resonant peak. Since we are exploring small system sizes, i.e., $N=12^{3}$ particles at $\rho=1$ and thus $L=12 \sigma$, this behavior turns out to be in apparent contradiction with some strategies recently introduced for probing the non-Debye spectrum, i.e., the so-called $\omega^{4}$ scaling, in models of disordered solids $[5,35,36]$.

Performing a detailed analysis of the density of states $D(\omega)$, at high parental temperatures, we observed that $D(\omega) \sim$ $\omega^{2}$ below $\omega_{0}$. The low-frequency tail changes to $D(\omega) \sim \omega^{4}$ as the temperature decreases toward $T_{d}$. The behavior of $F(\omega)$ confirms this finding.

Finally, we showed that our analysis of $D(\omega)$ is consistent with the behavior of $S(q, \omega)$ computed in the harmonic approximation. As has been observed in Ref. [32], $S(q, \omega) \rightarrow$ constant for $\omega \rightarrow 0$ whenever the inherent structures refer to high parental temperature configurations. When the parental temperature approaches $T_{d}, S(q, \omega \rightarrow 0) \sim \omega^{2}$, this finding supports a scaling $D(\omega) \sim \omega^{4}$.

In conclusion, our work suggests that soft-extended modes are responsible for Debye's scaling at frequencies lower than $\omega_{0}$, i.e., the lowest resonant frequency of $S_{T}(q, \omega)$, and high parental temperatures. As discussed in Ref. [9], this fact gives rise to a crossover in the low-frequency tail of $F(\omega)$ that changes its slope smoothly from Debye's law; i.e., the vibrational DOS changes from $D(\omega) \sim \omega^{2}$ to the famous $\omega^{4}$ scaling, by lowering the parental temperature. The results presented here confirm that the crossover is related to the suppression of extended modes below the lowest transverse mode.

The distribution $\mathcal{P}(\mathcal{R})$ of the inverse of the participation ratio $\mathcal{R}$ suggests that (i) the statistical properties of normal modes around the resonant peak of $S_{T}(q, \omega)$ show a weak dependency on parental temperature, and (ii) the same properties at lower frequencies turn out to be parental temperature dependent. The results presented here suggest that small system sizes are particularly suitable for probing these extended nonphononic modes. Further investigations are required for understanding the role played by these modes in the thermal and transport properties of structural glasses [37].

Considering our results in connection with those in Refs. $[5,36,38]$, it turns out that annealing protocols play a pivotal role in detecting deviations from Debye's law, as has been mentioned in Ref. [27].

Our study also suggests that to collect information about low-frequency extended modes, it turns to be much more convenient, in terms of computational time, to consider small system sizes instead of probing a larger system. This is because typical diagonalization algorithms scale with $N^{3}$ and thus, considering smaller system sizes $M=N / n$, with $n>1$, the same information is collected in a time that scales as $N^{3} / n^{2}$. On the other hand, larger system sizes result in being more suitable for investigating the spatial properties of extended and localized modes [6,37].

\section{ACKNOWLEDGMENTS}

We thank Gabriele Sicuro for critical readings of this manuscript. G.P. acknowledges the financial support of the Simons Foundation (Grant No. 454949). This work was also supported by the Joint Laboratory on "Advanced and Innovative Materials," ADINMAT, WISSapienza (G.P. and M.P.). M.P. and L.A. acknowledge funding from Regione Lazio, Grant Prot. No. 85-2017-15257 ("Progetti di Gruppi di Ricerca - Legge 13/2008 - Art. 4"). M.P. is supported by the H2020 program and by the Secretary of Universities and Research of the Government of Catalonia through Beatriu de Pinós program Grant No. 2018 BP 00088.
[1] D. Khomenko, C. Scalliet, L. Berthier, D. R. Reichman, and F. Zamponi, Depletion of Two-Level Systems in Ultrastable Computer-Generated Glasses, Phys. Rev. Lett. 124, 225901 (2020).
[2] U. Buchenau, Y. M. Galperin, V. L. Gurevich, and H. R. Schober, Anharmonic potentials and vibrational localization in glasses, Phys. Rev. B 43, 5039 (1991). 
[3] V. Gurarie and J. T. Chalker, Bosonic excitations in random media, Phys. Rev. B 68, 134207 (2003).

[4] M. Baity-Jesi, V. Martín-Mayor, G. Parisi, and S. Perez-Gaviro, Soft Modes, Localization, and Two-Level Systems in Spin Glasses, Phys. Rev. Lett. 115, 267205 (2015).

[5] E. Lerner, G. Düring, and E. Bouchbinder, Statistics and Properties of Low-Frequency Vibrational Modes in Structural Glasses, Phys. Rev. Lett. 117, 035501 (2016).

[6] H. Mizuno, H. Shiba, and A. Ikeda, Continuum limit of the vibrational properties of amorphous solids, Proc. Natl. Acad. Sci. USA 114, E9767 (2017).

[7] L. Angelani, M. Paoluzzi, G. Parisi, and G. Ruocco, Probing the non-Debye low-frequency excitations in glasses through random pinning, Proc. Natl. Acad. Sci. USA 115, 8700 (2018).

[8] L. Wang, A. Ninarello, P. Guan, L. Berthier, G. Szamel, and E. Flenner, Low-frequency vibrational modes of stable glasses, Nat. Commun. 10, 26 (2019).

[9] M. Paoluzzi, L. Angelani, G. Parisi, and G. Ruocco, Relation between Heterogeneous Frozen Regions in Supercooled Liquids and Non-Debye Spectrum in the Corresponding Glasses, Phys. Rev. Lett. 123, 155502 (2019).

[10] M. Ozawa, W. Kob, A. Ikeda, and K. Miyazaki, Equilibrium phase diagram of a randomly pinned glass-former, Proc. Natl. Acad. Sci. USA 112, 6914 (2015).

[11] C. Cammarota and G. Biroli, Ideal glass transitions by random pinning, Proc. Natl. Acad. Sci. USA 109, 8850 (2012).

[12] S. Karmakar and G. Parisi, Random pinning glass model, Proc. Natl. Acad. Sci. USA 110, 2752 (2013).

[13] K. Kim, Effects of pinned particles on the structural relaxation of supercooled liquids, Europhys. Lett. 61, 790 (2003).

[14] W. Kob and D. Coslovich, Nonlinear dynamic response of glass-forming liquids to random pinning, Phys. Rev. E 90, 052305 (2014).

[15] W. Schirmacher, G. Ruocco, and V. Mazzone, Heterogeneous Viscoelasticity: A Combined Theory of Dynamic and Elastic Heterogeneity, Phys. Rev. Lett. 115, 015901 (2015).

[16] W. Schirmacher, G. Ruocco, and V. Mazzone, Theory of heterogeneous viscoelasticity, Philos. Mag. 96, 620 (2016).

[17] W. Schirmacher, G. Diezemann, and C. Ganter, Harmonic Vibrational Excitations in Disordered Solids and the "Boson Peak", Phys. Rev. Lett. 81, 136 (1998).

[18] W. Schirmacher, Thermal conductivity of glassy materials and the "boson peak", Europhys. Lett. 73, 892 (2006).

[19] L. Berthier and G. Biroli, Theoretical perspective on the glass transition and amorphous materials, Rev. Mod. Phys. 83, 587 (2011).

[20] W. Kob, C. Donati, S. J. Plimpton, P. H. Poole, and S. C. Glotzer, Dynamical Heterogeneities in a Supercooled LennardJones Liquid, Phys. Rev. Lett. 79, 2827 (1997).

[21] N. Laāević, F. W. Starr, T. B. Schrøder, and S. C. Glotzer, Spatially heterogeneous dynamics investigated via a time- dependent four-point density correlation function, J. Chem. Phys. 119, 7372 (2003).

[22] G. Biroli, J.-P. Bouchaud, K. Miyazaki, and D. R. Reichman, Inhomogeneous Mode-Coupling Theory and Growing Dynamic Length in Supercooled Liquids, Phys. Rev. Lett. 97, 195701 (2006).

[23] G. Biroli and J.-P. Bouchaud, Diverging length scale and upper critical dimension in the mode-coupling theory of the glass transition, Europhys. Lett. 67, 21 (2004).

[24] E. Flenner, H. Staley, and G. Szamel, Universal Features of Dynamic Heterogeneity in Supercooled Liquids, Phys. Rev. Lett. 112, 097801 (2014).

[25] L. Berthier, G. Biroli, J.-P. Bouchaud, L. Cipelletti, and W. van Saarloos, Dynamical Heterogeneities in Glasses, Colloids, and Granular Media (Oxford University Press, Oxford, 2011).

[26] L. Berthier, Dynamic heterogeneity in amorphous materials, Physics 4, 42 (2011).

[27] E. Lerner, G. Düring, and E. Bouchbinder, Erratum: Statistics and Properties of Low-Frequency Vibrational Modes in Structural Glasses [Phys. Rev. Lett. 117, 035501 (2016)], Phys. Rev. Lett. 119, 099901(E) (2017).

[28] B. Bernu, J. P. Hansen, Y. Hiwatari, and G. Pastore, Soft-sphere model for the glass transition in binary alloys: Pair structure and self-diffusion, Phys. Rev. A 36, 4891 (1987).

[29] T. S. Grigera and G. Parisi, Fast Monte Carlo algorithm for supercooled soft spheres, Phys. Rev. E 63, 045102(R) (2001).

[30] J.-F. Bonnans, J. C. Gilbert, C. Lemaréchal, and C. A. Sagastizábal, Numerical Optimization: Theoretical and Practical Aspects (Springer Science \& Business Media, SpringerVerlag, Berlin, Heidelberg, New York, 2006).

[31] R. Bell and P. Dean, Atomic vibrations in vitreous silica, Discuss. Faraday Soc. 50, 55 (1970).

[32] V. Mazzacurati, G. Ruocco, and M. Sampoli, Low-frequency atomic motion in a model glass, Europhys. Lett. 34, 681 (1996).

[33] G. Monaco and S. Mossa, Anomalous properties of the acoustic excitations in glasses on the mesoscopic length scale, Proc. Natl. Acad. Sci. USA 106, 16907 (2009).

[34] A. Marruzzo, W. Schirmacher, A. Fratalocchi, and G. Ruocco, Heterogeneous shear elasticity of glasses: The origin of the boson peak, Sci. Rep. 3, 1407 (2013).

[35] E. Lerner and L. Gartner, Nonlinear modes disentangle glassy and Goldstone modes in structural glasses, SciPost Phys. 1, 016 (2016).

[36] E. Lerner and E. Bouchbinder, Effect of instantaneous and continuous quenches on the density of vibrational modes in model glasses, Phys. Rev. E 96, 020104(R) (2017).

[37] L. Wang, L. Berthier, E. Flenner, P. Guan, and G. Szamel, Sound attenuation in stable glasses, Soft Matter 15, 7018 (2019).

[38] G. Kapteijns, E. Bouchbinder, and E. Lerner, Universal Nonphononic Density of States in 2D, 3D, and 4D Glasses, Phys. Rev. Lett. 121, 055501 (2018). 